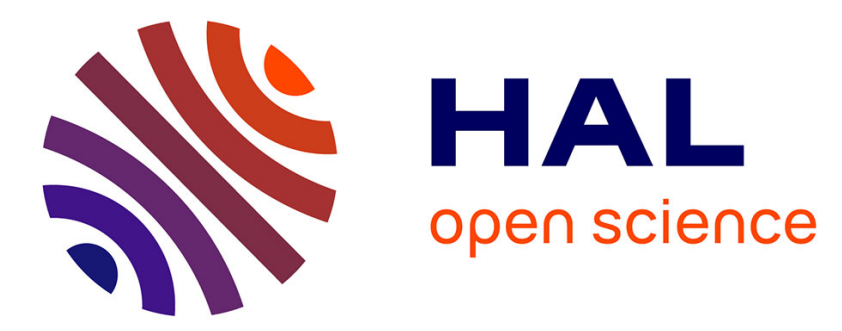

\title{
Modeling User Performance on Curved Constrained Paths
}

\author{
Mathieu Nancel, Edward Lank
}

\section{To cite this version:}

Mathieu Nancel, Edward Lank. Modeling User Performance on Curved Constrained Paths. ACM CHI 2017 - ACM Conference on Human Factors in Computing Systems, May 2017, Denver, United States. 10.1145/3025453.3025951. hal-01523455

\section{HAL Id: hal-01523455 \\ https://hal.inria.fr/hal-01523455}

Submitted on 21 Jun 2017

HAL is a multi-disciplinary open access archive for the deposit and dissemination of scientific research documents, whether they are published or not. The documents may come from teaching and research institutions in France or abroad, or from public or private research centers.
L'archive ouverte pluridisciplinaire HAL, est destinée au dépôt et à la diffusion de documents scientifiques de niveau recherche, publiés ou non, émanant des établissements d'enseignement et de recherche français ou étrangers, des laboratoires publics ou privés. 


\title{
Modeling User Performance on Curved Constrained Paths
}

\author{
Mathieu Nancel ${ }^{1,2,4}$ \& Edward Lank ${ }^{1,3}$ \\ ${ }^{1}$ Cheriton School of Computer Science, University of Waterloo, Canada \\ ${ }^{2}$ Aalto University, Helsinki, Finland \\ ${ }^{3}$ University of Lille \& ${ }^{4}$ Inria Lille, France \\ mathieu.nancel@inria.fr, lank@uwaterloo.ca
}

\begin{abstract}
In 1997, Accot and Zhai presented seminal work analyzing the temporal cost and instantaneous speed profiles associated with movement along constrained paths. Their work posited and validated the steering law, which described the relationship between path constraint, path length and the temporal cost of path traversal using a computer input device (e.g. a mouse). In this paper, we argue that the steering law fails to correctly model constrained paths of varying, arbitrary curvature, propose a new form of the law that accommodates these curved paths, and empirically validate our model.
\end{abstract}

\section{ACM Classification Keywords}

H.5.2 User Interfaces

\section{Author Keywords}

Steering Law; Movement; Models.

\section{INTRODUCTION}

In Human-Computer Interaction (HCI) research, performance models, i.e. mathematical models that characterize the temporal or kinematic properties of actions, [1, 3, 6, 8, 15, 17], are a common area of inquiry. Models from psychology such as Fitts's Law [9] and the Hick Hyman Law [19] have been used to describe selection time (pointing or choice) in interfaces. HCI researchers have extended and leveraged these models in various ways: for example, models have been adapted to describe pointing time for bivariate targets [3], to characterize novice-to-expert transitions in interface manipulation [8], and to describe the behavior of pointing facilitation techniques [4]

In 1997, Jon Accot and Shumin Zhai [1, 2] developed a novel, temporal model that described the cost of traversing constrained paths, the steering model. The steering model relates the time taken to traverse a path to the length of the path and the tolerance, or width, of the path, i.e.

$$
t=a+b\left(\frac{A}{W}\right)
$$

Permission to make digital or hard copies of all or part of this work for personal or classroom use is granted without fee provided that copies are not made or distributed for profit or commercial advantage and that copies bear this notice and the full citation on the first page. Copyrights for components of this work owned by others than the author(s) must be honored. Abstracting with credit is permitted. To copy otherwise, or republish, to post on servers or to redistribute to lists, requires prior specific permission and/or a fee. Request permissions from Permissions@ acm.org.

CHI 2017, May 06 - 11, 2017, Denver, CO, USA

Copyright is held by the owner/author(s). Publication rights licensed to ACM. ACM 978-1-4503-4655-9/17/05âAe\$15.00

DOI: http://dx.doi.org/10.1145/3025453.3025951
Here, $A$ represents the length of the path and $W$ represents the width of the path. The constant $a$ represents additive factors in starting and stopping [15], and $b$ is the reciprocal of the index of performance, a measure of the effectiveness of the input device. Accot and Zhai demonstrate that the steering model effectively predicts the temporal cost of straight, circular and spiral paths.

While the steering model is an appropriate representation of straight and circular paths, one open question is whether it can be generalized to represent paths of varying curvature. As Accot and Zhai note in their work leveraging the Steering Model to analyze paths [2], "One dimension that may affect steering difficulty but not modeled yet in the steering law is tunnel shape." Past work in psychology $[12,22,21]$ and HCI [14] argues that variation in curvature has a non-linear effect on movement speed, and, by implication, time.

In this paper, we derive a generalization of the steering model designed to incorporate both variations in curvature and in width (or tolerance) that allows prediction of both the instantaneous speed and the overall temporal cost of paths of arbitrary width and curvature. Through our experiments we show high correlation with temporal cost and instantaneous speed for both touch and mouse input.

Is there call for a generalized steering model? We argue that there is, primarily because of the rise in popularity of touchscreen displays. Modern multi-touch tablets support two different input touches: taps and strokes. While unconstrained strokes have been effectively modeled, not every input stroke is unconstrained: In a cluttered display contacting other interface objects can cause movement or other disruptions that require rearrangement. From intelligent selection [14], to shape tracing [26], to difficulty measures in games [5], understanding movement models under constraint can be a useful tool to design interaction techniques or overall applications. As a result, we argue that the modeling of constrained, arbitrary paths represents a useful enhancement of our ability to model generalized input on computer displays.

The remainder of this paper is organized as follows. We first review the steering model formulation and related psychology and HCI work that models curved trajectories. Next we present a mathematical model of expected time for paths of arbitrary width/curvature combinations. We then describe our experiments and data, before concluding with a discussion of the implications of our work. 


\section{RELATED WORK}

\section{Steering Model}

When moving toward a target along an unconstrained trajectory, movement follows a ballistic profile [10]. When constrained by endpoint, the kinematics $[16,18]$ and temporal cost of movement is described by Fitts's Law [9]:

$$
t=a+b \log \left(\frac{A+W}{W}\right)
$$

In this expression, the logarithmic term is known as the index of difficulty, the index of performance is the reciprocal of $b[2$, 15], and the constant $a$ represents additive factors. Additive factors include initiation or planning time at the beginning of a movement and time at the end of a movement between target acquisition and task delimitation (e.g. the time it takes between stopping and clicking) [15].

Leveraging Fitts's Law, Accot and Zhai [1] derived a theoretical steering law by representing a constrained path as an infinite series of targeting tasks along the trajectory. Given an infinite set of targeting tasks, each individual distance, $A$, shrinks to a vanishingly small distance, $d s$, and, at each location, a constraint on the direction of movement is specified, $W(s)$. By integrating over the entire path, $S$, we are left with an expression of the form:

$$
t=a+b \int_{S} \log \left(\frac{d s}{W(s)}+1\right)
$$

Performing a Taylor expansion on the logarithmic term and noting that $W(s) \gg d s$, yields:

$$
t=a+b \int_{S} \frac{d s}{\ln 2 \times W(s)}+\varepsilon \cong a+\frac{b}{\ln 2} \int_{S} \frac{d s}{W(s)}
$$

For a tunnel of fixed width, $W(s)$ is constant, yielding the simplified equation in the introduction. As well, because $b$ and $b / \ln 2$ differ only by a constant factor $\ln 2$, without loss of generality we will replace $b / \ln 2$ by $b$, taking it as understood that it will represent the slope of the straight-line equation in $I D$ and that its reciprocal is a function of input device performance, i.e. $I P$.

Alongside their work analyzing time, Accot and Zhai also note that an instantaneous law for speed of motion can be derived simply by noting that time equals distance divided by speed, i.e.:

$$
t=a+\int_{S} \frac{d s}{v(s)}
$$

Moving constant $b$ into the integral, this implies that:

$$
v(s)=\frac{1}{b} W(s)=I P \times W(s)
$$

While the simplified form of the steering law in the introduction is intuitive and easily understood, it is also the case that the simplified form of the steering law presented in the introduction, Equation (1) derived from Equation (4) with constant width, becomes significantly more complex as aspects of the tunnel change. For example, for uniformly narrowing or widening tunnels, Equation (4) becomes:

$$
t=a+b \frac{A}{W_{L}-W_{0}} \ln \frac{W_{L}}{W_{0}}
$$

Here $W_{0}$ is the width at the beginning of the tunnel, and $W_{L}$ is the width at the end of the tunnel.

Accot and Zhai also begin an analysis of a generalized steering law for arbitrary paths, leveraging, in particular, spiral paths. Here, the equation for a spiral path is again more complex:

$$
t=a+b \int_{2 \pi}^{2 \pi(n+1)} \frac{\sqrt{(\theta+\omega)^{6}+9(\theta+\omega)^{4}}}{(\theta+2 \pi+\omega)^{3}-(\theta+\omega)^{3}} d \theta
$$

In this equation, the numerator represents the length of a widening spiral of $n$ loops, and the denominator represents the gradually widening width of the spiral. In summary, Equation (1) represents only one type of tunnel: a fixed width tunnel. Equations (7), (8), and, in the general case, Equation (4), represent a much more accurate view of the complexities inherent in any representation of temporal complexity of path.

\section{Challenges to the Steering Law}

More recently, even the theoretically satisfying aspects of Equation (4) has failed to fully account for irregular tunnels. Yamanaka and Miyashita [24, 25] examine the temporal complexity of narrowing and widening tunnels. They note that widening tunnels take significantly less time than narrowing tunnels and introduce a free weight, $k$, to correct the movement time as follows:

$$
t=a+b\left(\frac{A}{W_{L}-W_{0}} \ln \frac{W_{L}}{W_{0}}+\frac{A\left(W_{L}-W_{0}\right)}{k W_{L} W_{0}}\right)
$$

In their Discussion of their result, Yamanaka and Miyashita note that the addition of a free weight parameter is unsatisfactory, and that a more accurate analysis of user behavior in light of a feed-forward model of constraint might explain the variation one sees in widening versus narrowing tunnels. In support of this, they point to speed profiles through their narrowing tunnel paths that demonstrate that, approximately 100 pixels prior to the end of the tunnel, participants' speed levels out and then begins to increase such that, at the region of highest constraint, participant speed is significantly faster than it was earlier in the tunnel despite the fact that the tunnel has decreased in width ([24], Figures 13, 14).

Second, Equation 4 does not model curvature as a component of the model. This is not a significant problem if curvature is constant over a path, but does become a problem if curvature varies. To understand the effects of curvature on time, consider the instantaneous speed model by Accot and Zhai, Equation 6. This equation predicts that speed is a function of width. However, what happens if curvature varies? Does this have an impact on speed? Consider Figure 1, taken from work on sloppy selection by Lank and Saund [14]. If curvature were unimportant, then the speed (vertical axis) should be constant over the path through the oval, but it is not. As predicted by the steering law, Figure 1 shows that path width affects speed 


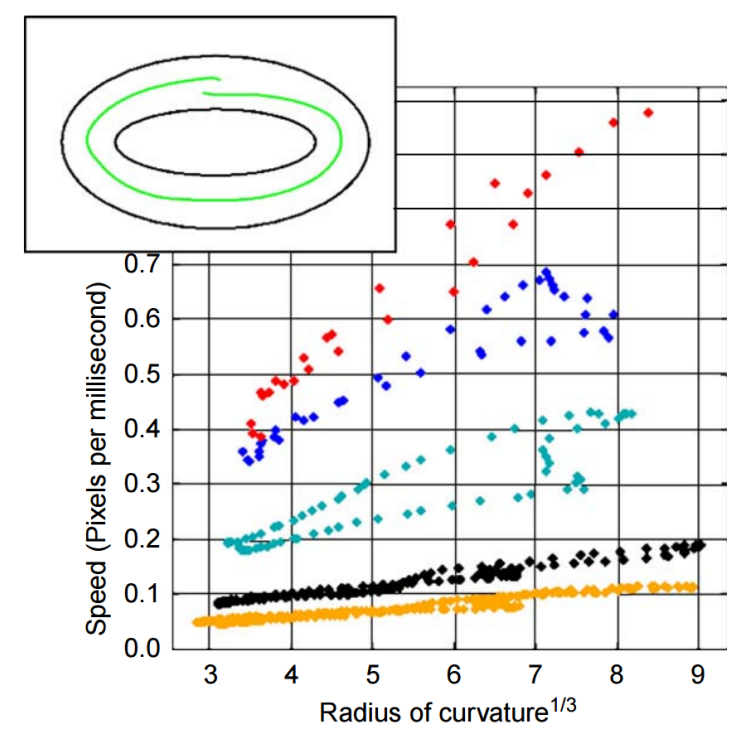

Figure 1. Speed profile of 10, 20, 40, 60, and 80-pixel constrained elliptical paths (from Lank \& Saund[10]).

in that narrower paths require users to go more slowly, but we also see an effect of varying curvature on speed: users move more slowly at locations of small radius of curvature and more rapidly at locations of high radius curvature (when the path is less curved).

Most of the work on the steering law has focused on time, not speed. However, we feel that it is important to understand both speed and time profiles for two reasons. First, variations in speed affect the time it takes to traverse a tunnel. If speed is related to tunnel width and tunnel curvature, then both of these effects combine to produce a cost model because time is simply distance divided by speed. Second, beyond the effect on cost models, a link between speed profiles and path constraints has implications for designing interaction techniques such as smart scissors, for the assessment of care in moving and drawing, and for difficulty measures in gaming, to name only a few examples. While Accot and Zhai only analyze straight-line, circular and spiral paths, they highlight the importance of a more generalized model for arbitrary paths in their work [2].

\section{Speed vs Curvature: The 2/3 Power Model}

One obvious conclusion that can be drawn from Lank and Saund's work on sloppy selection is that path width is insufficient to fully represent variations in speed, and therefore time, for the oval paths. As shown in Figure 1, there is a possible linear relationship between speed and the cube-root of radius of curvature alongside a linear increase in slope as a function of width or tolerance.

Lank and Saund draw inspiration for their analysis of trajectory models from studies of human performance in psychology. In this field, researchers note that curved trajectories typically follow a power law relationship, commonly called the $2 / 3$ power law [13, 22, 21] and formulated by Lacquaniti et al. [13] as follows:

$$
A(s)=\kappa[c(s)]^{2 / 3}
$$

In this equation, $A(s)$ represents the angular velocity in radians per second at point $\mathrm{s}=(\mathrm{x}, \mathrm{y})$ along a path, $c(s)$ is the curvature of the path at that point $s$ (the inverse of the radius of an arc that corresponds to the path), and $\kappa$ is a constant called the velocity gain factor ${ }^{1}$.

Our interest is not in angular velocity but in instantaneous speed. Transforming angular velocity, $A(s)$, to speed requires that we multiple angular velocity by the path radius, $R(s)$. Multiplying by radius at point $\mathrm{s}, R(s)$, and recognizing that curvature, $c(s)=1 / R(s)$, yields an equation for speed versus a path's radius of curvature, $R(s)$ :

$$
v(s)=\kappa[R(s)]^{1 / 3}
$$

One challenge with interpreting the $2 / 3$ power law is the velocity gain factor, $\kappa$. If instantaneous speed is measured in $\mathrm{m} / \mathrm{s}$, then $\kappa$ has units of $m^{2 / 3} / s$ [13]. While unintuitive, the unusual units are a result of the motor control processes that bias toward smooth movement [10] and of the complex link between speed and acceleration of a curved path in Cartesian coordinates and the path's radius of curvature [21]. Increasingly, researchers in HCI have leveraged the 2/3 power law relationship to model movement (e.g. $[6,14]$. However, beyond the work cited above on sloppy selection, we are unaware of any generalization of the steering model for paths of arbitrary curvature.

\section{FORMULATING A GENERALIZED STEERING MODEL}

Leveraging past work by Lank and Saund [14], Viviani and others [21], and Accot and Zhai [1], a straightforward formulation of a steering law for generalized paths would incorporate both aspects of path constraint and curvature, i.e.:

$$
t=a+\int_{S} \frac{d s}{v(s)}=a+b^{\prime} \int_{S} \frac{d s}{W(s) \times R(s)^{1 / 3}}
$$

Alongside this equation for the temporal cost of an arbitrary path, one can also calculate instantaneous speed along the path as:

$$
v(s)=\frac{1}{b^{\prime}} W(s) \times R(s)^{1 / 3}
$$

In this equation, the term $1 / b^{\prime}$ is the product of the index of performance (Equation (6) above) and the velocity gain factor, $\kappa$, from the $2 / 3$ power law, Equation (11). The term $W(s) R(s)^{1 / 3}$ is our model parameter, and we will call the value that this term takes model units for the remainder of this paper.

\footnotetext{
${ }^{1}$ In many formulations of the $2 / 3$ power law, there exists a parameter $\alpha=[0,0.1] . \alpha$ is a function of speed: for open-loop movement tasks such as scribbling, which are high speed, it's value approaches 0.1 . For careful drawing tasks - of the kind found in, for example, tunnel traversal, where speed is slow and movement control is closed loopits value $=0$, allowing us to ignore it. More information can be found in [21].
} 
Overall, at this point, we have two different hypothetical models to describe a generalized steering law. First, from Accot and Zhai, we have a model of the form of Equation (4), which incorporates only tunnel width and does not include curvature. Alongside this, we have a hypothesized model, Equation (12), which includes both tunnel width and curvature. We reproduce each of these models here, both for completeness and to simplify later contrasts for the reader.

Hypothetical Model 1 (Accot and Zhai, cf Equation 13 in [1]):

$$
t=a+b \int_{S} \frac{d s}{W(s)}
$$

Hypothetical Model 2 (proposed by this paper):

$$
t=a+b^{\prime} \int_{S} \frac{d s}{W(s) R(s)^{1 / 3}}
$$

Alongside these temporal models we have two speed models that correspond directly to instantaneous forms of these speed models. The two speed models are Equations (6) and (13), respectively:

Hypothetical Instantaneous Speed Model 1 (Accot and Zhai, cf Equation 20 in [1]):

$$
v(s)=\frac{1}{b} W(s)
$$

Hypothetical Instantaneous Speed Model 2 (proposed by this paper):

$$
v(s)=\frac{1}{b^{\prime}} W(s) \times R(s)^{1 / 3}
$$

\section{MODEL VALIDATION}

To contrast the above models, two options present themselves. First, one can leverage the total traversal time of a path to analyze whether the temporal cost model is correct, contrasting Equations (4) and (12). Alongside this, and more revealing, one can analyze the speed profiles, Equations (6) and (13). Our experiment evaluated the ability of the model to predict speed during constrained steering tasks on a touch input display. While we believe that a generalized model of steering under paths of varying curvature is most relevant to touch input, it is also important to evaluate modifications of the steering law using a mouse as an input device for experimental consistency with the original work on steering law by Accot and Zhai [1].

\section{Experimental Task}

Our experimental task was a steering task using paths depicted in Fig. 2-bottom. Paths were displayed on the screen in cyan over a black background. Participants, using a designated input device (touch or mouse), began movement outside the constrained path near the red half-circle shown at the bottom right, entered the path by crossing the red half-circle, and then were required to traverse the path without exiting from end indicated by the red half-circle to the unmarked end of the path. The paths were unidirectional as shown in Fig. 2-bottom, i.e. paths had their start and endpoint at opposite sides of the screen offset by arbitrary angles; these paths would typically not result in occlusion as their unidirectional nature would allow a participant to position him or herself such that their arm did not block the path during path traversal, reducing any confounds that repositioning to address occlusion [23] might create in the captured data.

\section{Apparatus}

The study was implemented in Java 7 and run on a first generation Microsoft Surface Pro tablet running Windows 8. The display is $23.4 \times 13.1 \mathrm{~cm}(208 \mathrm{DPI})$ with an input rate of $126 \mathrm{~Hz}$. Input was captured using both the multi-touch display of the Surface Pro (for touch modality) and using a generic, USB computer mouse (for mouse modality).

\section{Participants}

12 participants (7 males, mean age 28) participated in the experiment. All were right handed.

\section{Procedure}

The experiment was completed in two blocks, with at least one week between blocks to prevent learning effects. In the first block, all participants completed the task using the multi-touch display with their finger as the input device. In the second block, six of the participants returned and repeated the same paths using the mouse as the input device.

For each block, participants traversed 11 constrained paths. Each block of the experiment proceeded as follows. First, participants were introduced to (or reminded of) the steering task. Similar to directives provided to participants in Fitts's Law tasks, participants were asked to drag the input device inside the path as fast as they could without moving outside of the path. A red half-disc represented the beginning of the path, the point at which they had to start. All participants were presented with the same set of paths. The order of the paths was randomized, and the first two paths were considered training and not logged.

To allow users to validate their movements, the trace of the finger's actual movement was represented as a blue line on the display. If the participant crossed the path boundary, the trace would turn red; this was considered an error and, when it happened, a 'Restart' button was shown and participants were instructed to tap it to restart the trial. Participants were given three attempts to successfully complete a path, after which the next trial started. If any path was not completed successfully within three attempts, that path was presented a final time at the end of the study for a single, final attempt. The study lasted about 20 minutes for each participant.

\section{Path Generation}

The 11 paths were generated randomly before the study began in four steps. The process for generating paths is shown in Figure 3, and occurs as follows:

1. A guiding line is defined crossing the center of the screen and starting from a random point located 5 to $15 \mathrm{~mm}$ away from the lateral edges, and at least $5 \mathrm{~mm}$ away from the top and bottom edges (Fig. 2-a).

2. A set of $N$ points (6 to 10 ) is defined along the guide, with random distances between them, and with uniform noise 

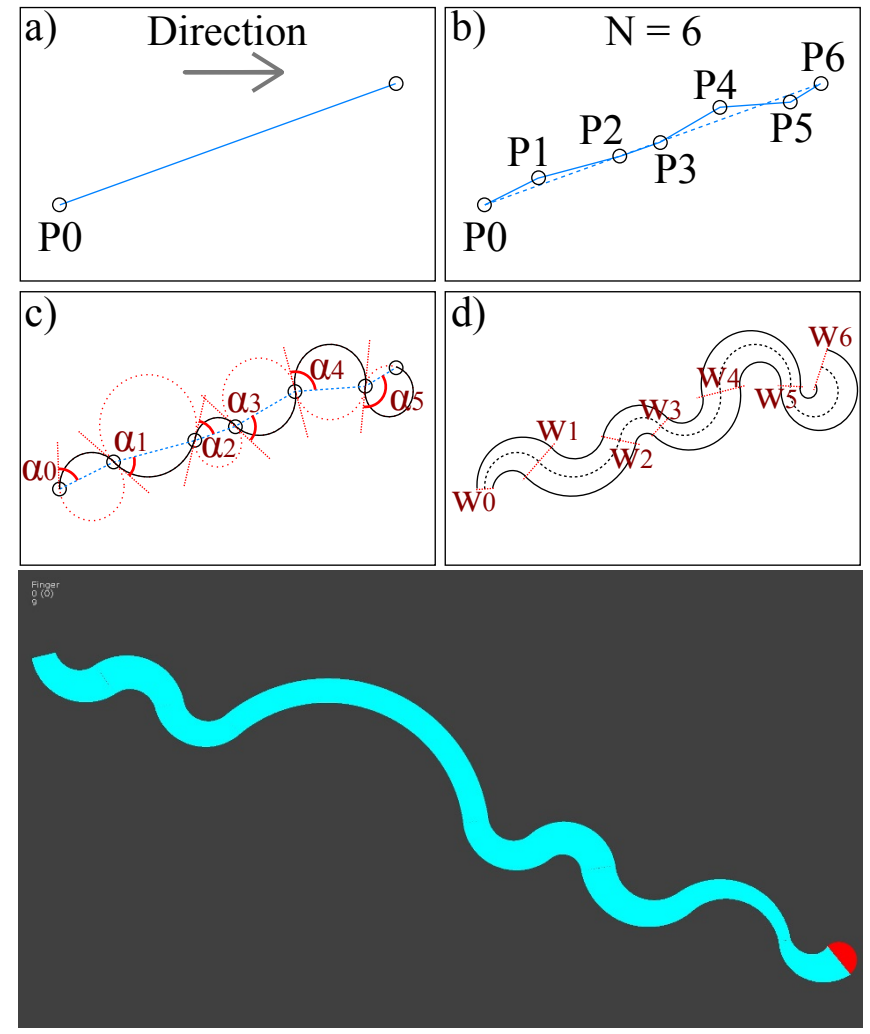

Figure 2. Creating a linear path from $P_{0}$ to $P_{6}$, modeled as 6 arcs with 6 smoothly varying width constraints. At the bottom, a path from the experiment.

$([-1,1] \mathrm{mm}$ ) added to their coordinates (Fig. 2-b) to offset them from the guiding line in step 1.

3. The actual trajectory is defined. An initial angle $\alpha_{0}$ is chosen randomly within $[-\pi / 2.5,-\pi / 5] \cup[\pi / 5, \pi / 2.5]$ radians at the defined starting point $P_{0}$ (left- or rightmost) and an arc is generated from the starting point to the next point $P_{1}$, its center equidistant from $P_{0}$ and $P_{1}$ and its tangent at $P_{0}$ matching $\alpha_{0}$. The tangential angle $\alpha_{1}$ of this arc at $P_{1}$ is then used to compute the next arc to $P_{2}$, and so on until $P_{N}$ is reached (Fig. 2-c).

4. The final path is generated by randomly selecting widths for every point $P_{n}$ (within $[3,13] \mathrm{mm}$ for the width for each point, $P_{n}$, and interpolating width to width variation linearly between $P_{n}$ and $P_{n+1}$ (Fig. 2-d).

Our measurements above result in path widths that vary between $3 \mathrm{~mm}$ and $13 \mathrm{~mm}$ and radii of curvature that vary between $6 \mathrm{~mm}$ and $41 \mathrm{~mm}$. Considering width, in the original experiment of Accot and Zhai [1], pixel measurements were used to describe tunnel width, not mm. Our selection of path size was inspired by results in Accot and Zhai's work on input device performance, in particular their data (see Figure 9 in [2]) which shows nonlinearity effects that appear in mouse/touchpad/stylus data at 70 pixels in path width on their hardware configurations (i.e. just under $18 \mathrm{~mm}$ ). While we use touch and mouse, not touchpad, wider path non-linearity [2] might impact the performance of models, so we wanted to be below the 70 pixel cut-off they used. Our path widths correspond to pixel widths of 10 to 50 pixels on Accot and Zhai's hardware configuration [1].

\section{Speed Calculation and Model Parameter Specification}

For each sample, we capture position time data over the course of the user's trajectory. Position is in screen coordinates (x, y) in millimeters, and time is captured in milliseconds. Given this initial data, our goal is to plot speed against model parameters of width (Eq. 6) and width $\times$ cube-root of radius of curvature (Eq. 13).

To calculate speed from participant input data, we perform a second degree polynomial regression of $\mathrm{x}(\mathrm{t})$ and $\mathrm{y}(\mathrm{t})$ data as described by Lank and Saund [14] at each input point on each path for each participant. To calculate speed in this fashion, we first had to decide how many data points to include in the regressions. Calculating speed and acceleration using three data points results in a signal containing a significant amount of sampling noise, while using too many points results in oversmoothing. We analyzed second-order polynomial regressions for a range of time-window sizes ( 0 to $4 \mathrm{~s}$ ), shown in Figure 3 on the vertical axis. Using a window of $1200 \mathrm{~ms}$, we find that we can eliminate high frequency noise that results from neurophysiological factors $[11,20]$ and sampling idiosyncrasies [14] while still preserving speed fluctuations on the order of $100 \mathrm{~ms}(10 \mathrm{~Hz})$.

\section{Research Questions}

There are two primary research questions that we explore with our study.

First, given recent work on narrowing versus widening tunnels, and, in particular, observations from Yamanaka and Miyashita $[24,25]$ on feedforward modeling of movement (see figures 13 and 14 from [24]), we first explore whether or not our participants pre-plan, and what that window pre- planning is. We expect that the free weight value is a direct result of path planning; if one examines the derivation of the free weight parameter in Yamanaka and Miyashita, they use path segments of short length to define an ID modifier, $I D_{\text {gap }}$, for their tunnel segments. The question then becomes: What is the length of this short segment?

Second, an open question is which model correlates better with observed movement data, Equation (6) or Equation (13). To address this question, we perform a fit over both models and analyze the results.

\section{RESULTS}

\section{Result 1: Do Users Pre-Plan?}

Given our data, there exists an initial question motivated by Yamanaka and Miyashita: At what point do width and radius of curvature constrain speed? Is it the current point, i.e. motion planning results in near perfect coordination of constraint and movement? Or is it some point slightly ahead of movement (in the future), i.e. the user anticipates the oncoming constraint and plans ahead? 


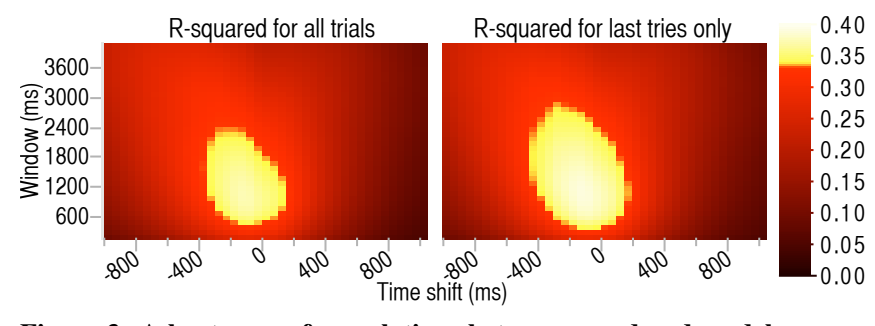

Figure 3. A heat map of correlations between speed and model parameters. We find that participants plan approximately $100 \mathrm{~ms}$ into the future, i.e. about $0.1 \mathrm{~s}$.

To perform this analysis, we use exactly the same technique as Yamanaka and Miyashita leverage in their paper, namely we explore $r^{2}$ values over time and space.

Figure 3 depicts this analysis along the horizontal axis. Analyzing the linear fit of speed against model values, we found that correlation is maximized if we consider constraint from a point $100 \mathrm{~ms}$ and $200 \mathrm{~ms}$ into the future. There is no difference in the minimization function for either model. Essentially, from our data it appears that, for steering tasks, reaction to constraint occurs on a time interval of approximately 0.1-0.2 s prior to the constraint. This value correlates well with past work in motor planning $[16,18,20]$.

Alongside temporal pre-planning, it is also possible that preplanning occurs over a fixed distance versus a fixed time interval. In analyzing movement, we find that pre-planning distance varies. For narrower tunnels, participants' pre-planning distance is shorter than for wider tunnels, and this distance seems to correlate with on-coming width. Given the linear relationship between width and speed predicted by both models, it seems clear that users pre-plan over a temporal interval, i.e. the pre-planning is predicated on the need to have time to react to impending constraint. We use a pre-planning interval of 150 ms during our analysis.

\section{Result 2: Model Correlation}

Any movement data will have significant neurophysiological noise. The standard approach to this, typified by analysis in Fitts's Law experiments, is to average speed values over a large set of data points for multiple users to produce an overall model of time against ID values for movement [7] Averaging is challenging with data when the independent variable is continuous. In typical Fitts's Law laboratory experiments, because discrete values are used for ID, averaging is simplified. In real-world pointing tasks where ID varies continuously, averaging must be handled differently.

To average our data points, we used a technique inspired by Chapuis et al. [7] in their analysis of real world Fitts-style movement data. We binned data into ranges for width and ranges for radius of curvature, specifically, in our case, 10 different width bins and 3 different radius of curvature bins, yielding 30 data bins overall. We chose these bins because 10 different bins of width corresponds to $1 \mathrm{~mm}$ per bin width (width varies from $3 \mathrm{~mm}$ to $13 \mathrm{~mm}$ ). Radius of curvature ranges from $6 \mathrm{~mm}$ to $41 \mathrm{~mm}$; when taking the cube root, this yields numerical values between 1.8 and 3.4 .

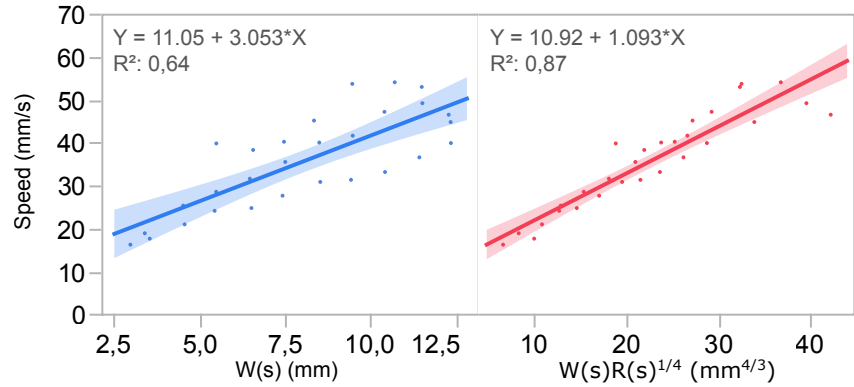

Figure 4. Plot of binned data values for Accot and Zhai (left) and for our model (right) on 30 data points for touch data. Note that incorporating curvature data results in much less variability in data with respect to the straight-line fit.

To replicate Fitts's style analysis, we calculated an average (model unit, speed) value for each data bin and an average (width, speed) data point for each bin for each participant. Similar to Fitts's Law analysis [4, 7], we also took a "mean-ofmeans" measure, i.e. the average of participant values for each bin to create an overall best-fit line. Once averaged by participant and overall, we treated all data values identically; this is commonly done in Fitts-style analysis [7], where average time is plotted in unweighted form against ID and correlations are typically performed treating all averaged data points with equal weight.

To determine which model most accurately represents data, two options present themselves: either the radius of curvature term will improve the correlation, $r^{2}$, or it will not. We performed a linear fit of the data using both model parameters (proposed by this paper) and width values (Accot and Zhai's model) on the 30 averaged data points per user and averaged across all users. For our model, data points were $\left(W(s) R(s)^{1 / 3}, v(s)\right)$ averaged per bin; for Accot and Zhai, data points were $(W(s), v(s))$ values again averaged per bin.

\section{Touch Data}

Table 1 depicts $r^{2}$ values for the model proposed by this paper and for the Accot and Zhai model using our touch input data.

\begin{tabular}{c|rrr|rrr} 
& \multicolumn{3}{|r|}{ Accot and Zhai } & \multicolumn{3}{|c}{ Proposed Model } \\
& $a$ & $b^{\prime}$ & $r^{2}$ & $a$ & $b$ & $r^{2}$ \\
\hline P1 & 4.29 & 3.76 & .61 & 4.62 & 1.32 & .80 \\
P2 & 3.80 & 5.85 & .77 & 9.34 & 1.84 & .81 \\
P3 & 24.65 & 2.28 & .35 & 24.37 & 0.83 & .49 \\
P4 & 10.80 & 3.65 & .71 & 12.82 & 1.22 & .82 \\
P5 & 11.80 & 4.16 & .39 & 4.24 & 1.80 & .78 \\
P6 & 7.23 & 2.50 & .40 & 4.74 & 0.99 & .67 \\
P7 & 2.80 & 3.76 & .56 & 0.92 & 1.42 & .84 \\
P8 & 4.25 & 2.99 & .71 & 4.63 & 1.05 & .91 \\
P9 & 27.42 & 2.54 & .47 & 27.09 & 0.91 & .65 \\
P10 & 27.15 & 1.91 & .41 & 27.89 & 0.65 & .50 \\
P11 & 21.95 & 1.72 & .37 & 21.31 & 0.64 & .54 \\
P12 & 13.26 & 2.24 & .46 & 12.99 & 0.81 & .63 \\
\hline Overall & 11.05 & 3.05 & .64 & 10.92 & 1.09 & .87
\end{tabular}

Table 1. Comparison of coefficients ( $a$ or y-intercept and $b^{\prime} / b$ or slope) and correlation $\left(r^{2}\right)$ for our model and for Accot and Zhai's model for touch data. 
Overall correlations of 0.87 and 0.64 are both statistically significant on 30 data points (for our model, $r^{2}=0.87, p<$ 0.0001; for Accot and Zhai, $r^{2}=0.64, p<0.0001$ ). This is unsurprising; width is an important component of both models. However, given two models, both with statistically significant correlations, a higher $r^{2}$ value represents a more encompassing model, i.e. our model accounts for more of the variability present in the data (basically $87 \%$ for our model versus $64 \%$ for theirs). Measuring whether our model represents a significant improvement on the data can be done using pairwise $\mathrm{t}$-tests of correlation values for each participant $(p<0.0001$ for pairwise, two-tailed t-test). The strong statistical signal is unsurprising, given an observation of the pairwise samples: for every participant, correlation improves for our model versus Accot and Zhai's model.

\section{Mouse Data}

While touch data provides a strong indication of the advantage of our model, one question we had was whether touch input might differ from mouse-based input, i.e. it is important to check that input device characteristics were not unfairly biasing against Accot and Zhai's model. Using the same method described above to produce Fitts's style data points, we performed an analysis of six of our study participants on mouse data. Table 2 depicts correlations for both our model and Accot and Zhai's model on mouse data. Similar to touch data, our model significantly outperforms Accot and Zhai both per user and overall (our model's $r^{2}=0.96$ vs Accot and zhai's model $r^{2}=0.77$ ).

\begin{tabular}{c|rrr|rrr} 
& \multicolumn{3}{|c|}{ Accot and Zhai } & \multicolumn{3}{|c}{ Proposed Model } \\
& $a$ & $b^{\prime}$ & $r^{2}$ & $a$ & $b$ & $r^{2}$ \\
\hline P1 & 6.96 & 2.13 & .84 & 9.31 & 0.65 & .84 \\
P2 & 11.21 & 3.38 & .62 & 10.24 & 1.24 & .88 \\
P3 & -1.17 & 4.32 & .72 & -0.59 & 1.50 & .93 \\
P6 & 5.86 & 2.09 & .72 & 6.85 & 0.70 & .85 \\
P7 & -0.32 & 2.61 & .73 & 0.20 & 0.91 & .91 \\
P8 & 4.82 & 2.23 & .67 & 4.77 & 0.80 & .88 \\
\hline Overall & 4.02 & 2.75 & .77 & 4.71 & 0.95 & .96
\end{tabular}

Table 2. Comparison of correlation coefficients, $r^{2}$, for our model and for Accot and Zhai's model for mouse data. Note, again, the significant reduction in variability with respect to the best-fit line.

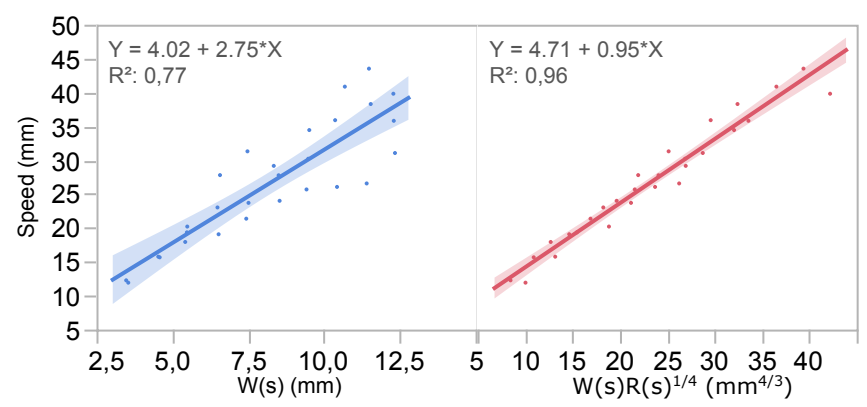

Figure 5. Mouse data plot of best-fit line (mean-of-means) for Accot and Zhai (left) and our model (right). Note, again, the reduction in variability when radius of curvature is represented in the model.

\section{Width Effects and Touch Data}

In our analysis of Accot and Zhai's original work, one issue that came to the fore was the discontinuities in path constraint.
Accot and Zhai note that their model only holds for path widths of less than 80 pixels on a $1024 \times 768$ pixel display. Furthermore, as we noted earlier, if one examines Figure 9 in Accot and Zhai, 1999 [2], Accot and Zhai note nonlinearity effects that become present in the data at values even as low as 70 pixels $(16 \mathrm{~mm}$ when converting from their experimental setup to SI units). The challenge is as follows (from the original Accot and Zhai steering law):

- For path widths of less than 80 pixels, Accot and Zhai propose the steering law.

- For unconstrained paths, Fitts's Law predicts a temporal cost.

- For path widths between 80 pixels and unconstrained (weakly constrained), a model discontinuity exists that has yet to be resolved. We are aware of no work that addresses the discontinuity between 80-pixel paths and unconstrained paths.

While mouse correlations are very high for our model, touch data has strong but slightly lower overall correlation values. To determine whether discontinuities were impacting the fit $\left(r^{2}\right)$ of our touch data, we analyzed lower model units (narrower curved paths) versus higher model units (wider curved paths) by segmenting the data into two halves, lower and higher values, along the $\mathrm{x}$-axis for each model. Table 3 shows the corresponding values for $r^{2}$ for both models for touch data. In this table, the first two rows represent a split using our model units and the second two rows represent a split using width, i.e. based on their model. Of significance in this table is correlation for Accot and Zhai's model at high model units/high widths, where their correlation is no longer statistically significant. Essentially, this indicates that for wider paths in our touch data, larger than $8 \mathrm{~mm}$, width is no longer a significant factor in performance. While our model also degrades, because curvature continues to affect speed, our model still retains some ability to explain variations in data. This trend is also apparent in Figure 4.

\begin{tabular}{c|c|c|c} 
Split & Model & $\begin{array}{c}\text { Low model } \\
\text { units } r^{2}\end{array}$ & $\begin{array}{c}\text { High model } \\
\text { units } r^{2}\end{array}$ \\
\hline \multirow{2}{*}{$W(s) R(s)^{1 / 3}$} & Our model & $\mathbf{0 . 8 5}$ & 0.36 \\
& Accot and Zhai & 0.48 & $0.00(\mathrm{~ns})$ \\
\hline$W(s)$ & Our model & $\mathbf{0 . 9 1}$ & 0.62 \\
& Accot and Zhai & 0.60 & 0.08 (ns)
\end{tabular}

Table 3. Examining correlation coefficients for lower-model-values and for higher-model-values for hypothesized model and Accot and Zhai with touch data. Note that, as expected, for weakly constrained paths, Accot and Zhai's model does not correlate with movement time or speed.

Examining high versus low values for our model and for width for mouse data (see Table 4 we find that width continues to have some affect on behavior for high model units (though $r^{2}$ values for our model continue to outperform their correlations).

\section{Error Effects}

In our experimental method, participants were given three successive attempts to successfully traverse a path, plus one 


\begin{tabular}{c|c|c|c} 
Split & Model & $\begin{array}{c}\text { Low model } \\
\text { units } r^{2}\end{array}$ & $\begin{array}{c}\text { High model } \\
\text { units } r^{2}\end{array}$ \\
\hline$W(s) R(s)^{1 / 3}$ & Our model & $\mathbf{0 . 9 4}$ & 0.79 \\
& Accot and Zhai & 0.76 & $0.17(\mathrm{~ns})$ \\
\hline$W(s)$ & Our model & $\mathbf{0 . 9 4}$ & 0.89 \\
& Accot and Zhai & 0.75 & 0.33
\end{tabular}

Table 4. Examining correlation coefficients for lower-model-values and for higher-model-values for hypothesized model and Accot and Zhai with mouse data. Note that, as expected, for weakly constrained paths, Accot and Zhai's model does not correlate with movement time or speed.

additional attempt at the end of the study if they failed three times. We hypothesized that multiple errors, each requiring a user to restart a given path, might result in users becoming ever-more careful, which, in turn, might result in changes to kinematic properties of movement. If kinematics change, that change might invalidate any model. We therefore ran our analyses with two path sets:

1. All: Analyze all paths generated by participant.

2. Last: Only the last attempt for a given path for each participant is analyzed (regardless of success or failure).

Table 5 depicts the $r^{2}$ values for model fits for both all and last. Correlations were virtually identical for All and Last, leading us to conclude that errors did not affect the performance of our model in steering tasks.

\begin{tabular}{l|cc|cc|}
\multirow{2}{*}{ Errors } & \multicolumn{2}{|c|}{ Touch } & \multicolumn{2}{c}{ Mouse } \\
& $r^{2}$ & $p$ & $r^{2}$ & $p$ \\
\hline All & 0.87 & $<0.0001$ & .96 & $<0.0001$ \\
Last & 0.86 & $<0.0001$ & .94 & $<0.0001$
\end{tabular}

Table 5. Correlation and significance for average movement speeds taking all paths versus last attempts.

\section{Towards an Instantaneous Model}

One aspect of our model (and of Accot and Zhai's speed versus constraint model) that is interesting to consider is its ability to serve as a real-time model, i.e. to explore how well the competing models fit every available data point. This would be akin to measuring correlation between index-ofdifficult and time for every single discrete pointing task in a Fitts's Law experiment, rather than taking user means and then mean of user means. Given the amount of neurophysiological noise in target acquisition tasks, one would expect very low correlations [7]. Table 6 shows these correlations and Figure 6 plots all data for both models. The model incorporating curvature outperforms Accot and Zhai's width-only model in all cases.

\section{DISCUSSION}

\section{Model Comparison}

We observe significantly higher correlation between our model, that incorporates curvature, compared to Accot and Zhai's.

The one challenge with our model and with Accot and Zhai's model is the discontinuities that exist between models and unconstrained movement. There are also discontinuities possible when individual constraints are adjusted instantaneously,

\begin{tabular}{c|rrr|rrr} 
& \multicolumn{3}{|c|}{ Accot and Zhai } & \multicolumn{3}{|c}{ Proposed Model } \\
& $a$ & $b^{\prime}$ & $r^{2}$ & $a$ & $b$ & $r^{2}$ \\
\hline P1 & 4.99 & 3.03 & .25 & 2.42 & 1.34 & .35 \\
P2 & -0.16 & 5.99 & .59 & 2.74 & 2.15 & .62 \\
P3 & 22.28 & 2.11 & .16 & 19.16 & 1.00 & .28 \\
P4 & 16.90 & 2.64 & .23 & 15.04 & 1.12 & .34 \\
P5 & 11.53 & 3.02 & .28 & 7.29 & 1.45 & .43 \\
P6 & 5.70 & 1.94 & .27 & 3.58 & 0.89 & .41 \\
P7 & 2.08 & 3.19 & .40 & -0.35 & 1.40 & .56 \\
P8 & 3.31 & 2.86 & .43 & 3.28 & 1.10 & .50 \\
P9 & 26.52 & 2.29 & .10 & 24.55 & 0.98 & .16 \\
P10 & 25.60 & 1.80 & .18 & 24.34 & 0.77 & .25 \\
P11 & 18.81 & 1.83 & .20 & 17.98 & 0.75 & .29 \\
P12 & 11.66 & 2.08 & .24 & 11.29 & 0.83 & .31 \\
\hline Overall & 9.99 & 2.77 & 0.22 & 8.36 & 1.78 & 0.30
\end{tabular}

Table 6. Comparison of correlation coefficients, $r^{2}$, for our model and for Accot and Zhai's model for touch data on approximately 7000 data points per user and 82965 data points for overall data.

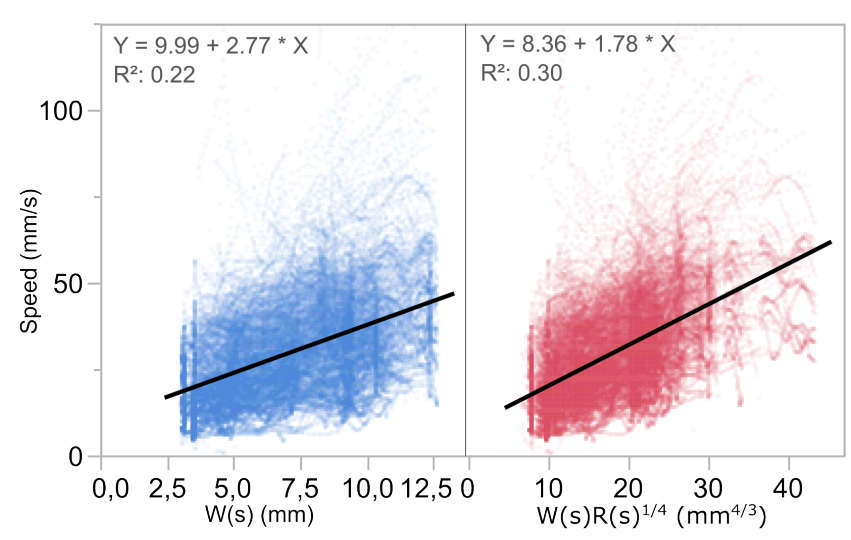

Figure 6. Plot of fitting parameters on all data points $(n=82965)$.

i.e. tunnel widths are defined by discontinuous values rather than varying smoothly over time. The closest model currently available to model movement between varying constraints is the minimum jerk model of goal directed movement [10]. The premise of this movement is that human motor control values movement that minimizes abrupt changes in acceleration. One could, therefore, model varying constraints through a piecewise function, such that, where constraints are continuous, users vary smoothly between constraints using constrained path models (Accot and Zhai for straight-line paths; ours for more arbitrary paths). Where discontinuities occur, users leverage minimum jerk speed adjustments to transition from one constraint to the next.

There exists some justification for a piecewise approach to aimed movement. Cao and Zhai [6] propose a temporal model of character formation that breaks pen strokes into three different substrokes: line segments, corners, and curves. A character consisting of a line segment and a curve segment, for example a ' $\mathrm{J}$ ' is modeled as a straight segment with a curved segment, and two different models define the temporal cost of the two different substrokes. 


\section{Trajectory Cost Models and Implications}

The steering law [1], as formulated by Accot and Zhai, was primarily considered a model of the temporal cost of path traversal, though, as they note, an instantaneous speed variant can easily be deduced. In our enhanced model of steering through irregular paths, our focus has, to this point, been primarily on the correlation between expected speed values at any specific point along the path and path parameters of width and radius of curvature.

Given the very highly statistically significant correlations between model parameters and instantaneous speed (all p-values $<0.001$ in experiments both on the tablet form factor and on the larger display with both touch and mouse input), it is obvious that the correlations that we hypothesize exist. Given the strength of the $r^{2}$ values between average speed across many trials at specific model points, it is also obvious that our model accurately characterizes the factors affecting expected speed.

One benefit of the speed model we provide is that it can easily be used either as a performance model to evaluate speed, a cost model to evaluate time, or a constraint model to evaluate perceived constraint for any given path. First, to model expected performance at a given point, our model accurately characterizes expected speed at that point. This satisfies the first implication, a characterization of the expected performance at any point along a trajectory.

To understand the use of this model to describe overall temporal cost, consider the relationship between speed, distance, and time commonly captured in introductory physics textbooks:

$$
v(s)=\frac{d s}{d t} \Longrightarrow d t=\frac{d s}{v(s)} \Longrightarrow t=c+\int \frac{d s}{v(s)}
$$

Thus, our model can easily be used to calculate expected cost of any arbitrary constrained path simply by evaluating the integral described above. If it proves impractical to calculate the integral, a summation can serve as an approximation, provided $\Delta s$ is set sufficiently small to ensure a good approximation to the integral:

$$
t=c+\int \frac{d s}{v(s)} \approx c+\sum \frac{\Delta s}{v(s)}
$$

Finally, as noted by Lank and Saund [14], a model that links speed to constraint and curvature can be used to infer the perceived constraint of a path being traversed by a user in a graphical user interface. Given the widespread adoption of multi-touch as an input modality, gestural input, both constrained and unconstrained is commonplace. From a gesture, speed and curvature can be calculated - the speed directly using numerical differentiation using an appropriate technique such as the polynomial regression described above and curvature (the inverse of radius of curvature) from parameters of movement via the formula:

$$
\text { curvature }=\frac{\left|v_{x} a_{y}-v_{y} a_{x}\right|}{\left(v_{x}^{2}+v_{y}^{2}\right)^{3 / 2}}
$$

The one challenge with an instantaneous model is that there exists significant neurophysiological noise in real-time input as we saw in Table 6, a result of users behavior deviating from the optimal due. These deviations are likely due to small errors in submovements $[16,18]$ and the need to iteratively correct those small errors. On the other hand, it is also the case that we do not necessarily need to predict instantaneous speed or infer instantaneous constraint at a pixel or millisecond level. If we group points together, perhaps over $10 \mathrm{~mm}$ or $20 \mathrm{~mm}$ of user movement, or we infer based on a slower sampling rate such as $5 \mathrm{~Hz}$ we can use averaged data points to infer overall constraint within a region, similar to a sliding window filter. An appropriate averaging window will be task-specific; techniques such as sloppy selection, intelligent scissors, or difficulty assessment for gaming tasks can select appropriate averaging intervals based on the fidelity of measure needed.

\section{CONCLUSION}

In this paper, we propose an extension of the original steering law work of Accot and Zhai [1], modifying it to take into account the effect of arbitrary variations of curvature along constrained paths. Our new formulation models the temporal cost of constrained path traversal via the following formula:

$$
t=a+b^{\prime} \int_{S} \frac{d s}{W(s) \times R(s)^{1 / 3}}
$$

We also provide a model of instantaneous speed of the form:

$$
v(s)=\frac{1}{b^{\prime}} W(s) \times R(s)^{1 / 3}
$$

We empirically validate this model and discuss its relationship to past work.

\section{ACKNOWLEDGMENTS}

This research underwent ethics review by the Office of Research Ethics at the University of Waterloo. Funding for this research was provided by the Natural Science and Engineering Research Council of Canada, by The European Research Council (ERC) Horizon 2020 Innovation and Research Program (Grant Agreement 637991) and by La Région Nord-Pas-deCalais, France.

\section{REFERENCES}

1. Johnny Accot and Shumin Zhai. 1997. Beyond Fitts' Law: Models for Trajectory-based HCI Tasks. In Proceedings of the ACM SIGCHI Conference on Human Factors in Computing Systems (CHI'97). ACM, New York, NY, USA, 295-302. DOI :

http://dx.doi.org/10.1145/258549.258760

2. Johnny Accot and Shumin Zhai. 1999. Performance Evaluation of Input Devices in Trajectory-based Tasks: An Application of the Steering Law. In Proceedings of the SIGCHI Conference on Human Factors in Computing Systems (CHI '99). ACM, New York, NY, USA, 466-472. DOI: http://dx.doi . org/10.1145/302979.303133

3. Johnny Accot and Shumin Zhai. 2003. Refining Fitts' Law Models for Bivariate Pointing. In Proceedings of the 
SIGCHI Conference on Human Factors in Computing Systems (CHI '03). ACM, New York, NY, USA, 193-200. DOI : http://dx.doi . org/10.1145/642611.642646

4. Ravin Balakrishnan. 2004. âĂIJBeatingâĂİ FittsâĂŹ law: virtual enhancements for pointing facilitation. International Journal of Human-Computer Studies 61, 6 (Dec. 2004), 857-874. DOI :

http: //dx.doi.org/10.1016/j.ijhcs. 2004.09.002

5. Daniel Boutros. 2008. Difficulty is Difficult: Designing for Hard Modes in Games. (2008).

http://www . gamasutra. com/view/feature/3787/difficulty

6. Xiang Cao and Shumin Zhai. 2007. Modeling Human Performance of Pen Stroke Gestures. In Proceedings of the SIGCHI Conference on Human Factors in Computing Systems (CHI '07). ACM, New York, NY, USA, 1495-1504. DOI :

http : //dx . doi .org/10.1145/1240624.1240850

7. Olivier Chapuis, Renaud Blanch, and Michel Beaudouin-Lafon. 2007. Fitts' Law in the Wild: A Field Study of Aimed Movements. Technical Report. https://hal . archives-ouvertes.fr/hal-00612026 LRI Technical Repport Number 1480, Univ. Paris-Sud, 11 pages.

8. Andy Cockburn, Carl Gutwin, and Saul Greenberg. 2007. A Predictive Model of Menu Performance. In Proceedings of the SIGCHI Conference on Human Factors in Computing Systems (CHI '07). ACM, New York, NY, USA, 627-636. DOI:

http://dx. doi . org/10.1145/1240624.1240723

9. Paul M. Fitts. 1954. The information capacity of the human motor system in controlling the amplitude of movement. Journal of Experimental Psychology 47, 6 (1954), 381-391. DOI :

http://dx. doi.org/10.1037/h0055392

10. T. Flash and N. Hogan. 1985. The coordination of arm movements: an experimentally confirmed mathematical model. Journal of Neuroscience 5, 7 (July 1985), 1688-1703. http://www. jneurosci . org/content/5/7/1688

11. D. M. Green and J. A. Swets. 1966. Signal detection theory and psychophysics. 1966. New York 888 (1966), 889.

12. P. L. Gribble and D. J. Ostry. 1996. Origins of the power law relation between movement velocity and curvature: modeling the effects of muscle mechanics and limb dynamics. Journal of Neurophysiology 76, 5 (Nov. 1996), 2853-2860. http://jn. physiology . org/content/76/5/2853

13. Francesco Lacquaniti, Carlo Terzuolo, and Paolo Viviani. 1983. The law relating the kinematic and figural aspects of drawing movements. Acta Psychologica 54, 1âĂŞ3 (Oct. 1983), 115-130. DOI : http : //dx . doi . org/10 . 1016/0001-6918(83)90027-6

14. Edward Lank and Eric Saund. 2005. Sloppy selection: Providing an accurate interpretation of imprecise selection gestures. Computers \& Graphics 29, 4 (Aug.
2005), 490-500. DOI :

http: //dx. doi .org/10.1016/j. cag. 2005.05.003

15. I. Scott MacKenzie. 1992. Fitts' Law As a Research and Design Tool in Human-computer Interaction. Hum.-Comput. Interact. 7, 1 (March 1992), 91-139. DOI : http://dx.doi .org/10.1207/s15327051hci0701_3

16. David E. Meyer, Richard A. Abrams, Sylvan Kornblum, Charles E. Wright, and J. E. 1988. Optimality in human motor performance: Ideal control of rapid aimed movements. Psychological Review 95, 3 (1988), 340-370. DOI : http: //dx . doi . org/10.1037/0033-295X. 95 . 3.340

17. Robert Pastel. 2006. Measuring the Difficulty of Steering Through Corners. In Proceedings of the SIGCHI Conference on Human Factors in Computing Systems (CHI '06). ACM, New York, NY, USA, 1087-1096. D0I : http://dx.doi . org/10.1145/1124772.1124934

18. Réjean Plamondon. 1995. A kinematic theory of rapid human movements. Biological Cybernetics 72, 4 (March 1995), 295-307. DOI :

http://dx.doi .org/10.1007/BF00202785

19. Steven C. Seow. 2005. Information Theoretic Models of HCI: A Comparison of the Hick-Hyman Law and Fitts' Law. Hum.-Comput. Interact. 20, 3 (Sept. 2005), 315-352. DOI :

http://dx.doi.org/10.1207/s15327051hci2003_3

20. Reza Shadmehr and Steven P. Wise. 2005. The computational neurobiology of reaching and pointing: a foundation for motor learning. MIT press.

21. Paolo Viviani and Tamar Flash. 1995. Minimum-jerk, two-thirds power law, and isochrony: converging approaches to movement planning. Journal of Experimental Psychology: Human Perception and Performance 21, 1 (1995), 32-53. DOI : http: //dx.doi . org/10.1037/0096-1523.21.1.32

22. Paolo Viviani and Roland Schneider. 1991. A developmental study of the relationship between geometry and kinematics in drawing movements. Journal of Experimental Psychology: Human Perception and Performance 17, 1 (1991), 198-218. DOI : http://dx.doi.org/10.1037/0096-1523.17.1.198

23. Daniel Vogel and Ravin Balakrishnan. 2010. Occlusion-aware Interfaces. In Proceedings of the SIGCHI Conference on Human Factors in Computing Systems (CHI '10). ACM, New York, NY, USA, 263-272. DOI : http://dx.doi.org/10.1145/1753326.1753365

24. Shota Yamanaka and Homei Miyashita. 2016a. Modeling the Steering Time Difference Between Narrowing and Widening Tunnels. In Proceedings of the 2016 CHI Conference on Human Factors in Computing Systems (CHI '16). ACM, New York, NY, USA, 1846-1856. DOI : http://dx.doi .org/10.1145/2858036.2858037

25. Shota Yamanaka and Homei Miyashita. 2016b. Scale Effects in the Steering Time Difference Between Narrowing and Widening Linear Tunnels. In Proceedings 
of the 9th Nordic Conference on Human-Computer

Interaction (NordiCHI '16). ACM, New York, NY, USA, 12:1-12:10. DOI :

http://dx. doi . org/10.1145/2971485.2971486

26. Stanislaw Zabramski, Suman Shrestha, and Wolfgang Stuerzlinger. 2013. Easy vs. Tricky: The Shape Effect in Tracing, Selecting, and Steering With Mouse, Stylus, and Touch. In Proceedings of International Conference on Making Sense of Converging Media (AcademicMindTrek '13). ACM, New York, NY, USA, 99:99-99:103. DOI :

http://dx. doi .org/10.1145/2523429.2523444 